\title{
Influence of Regenerator Thermal Mass Ratio on Stirling Engine Efficiency
}

\author{
Anders S. Nielsen, Brayden T. York, Brendan D. MacDonald \\ University of Ontario Institute of Technology \\ 2000 Simcoe Street North, L1H 7K4, Oshawa, Canada \\ anders.nielsen@uoit.net; brayden.york@uoit.net; brendan.macdonald@uoit.ca
}

\section{Extended Abstract}

Stirling engines are external combustion engines that have the ability to rely on an assortment of alternative and sustainable fuel sources, therefore making them ideal for clean energy production. They also have the potential to attain the highest theoretical engine efficiency due to the use of a regenerator, which stores thermal energy between the engine's expansion and compression strokes. Accordingly, regenerator design and development has garnered much attention in past research, and become a focus area for improving the overall performance and efficiency of Stirling engines. Regenerators are required to have a high heat transfer capacity, sufficient thermal storage capacity, while also limiting the resultant pressure drop as the working fluid flows through it. An interesting question that arises in the design of regenerators is how much thermal mass they should have in comparison to that of the working fluid, also known as the regenerator thermal mass ratio. The regenerator thermal mass ratio is an indicator of the regenerator's size, and an excessive amount of material will produce a higher resultant pressure drop, causing a decrease in Stirling engine performance. It is therefore desirable to select a value of regenerator thermal mass ratio that minimizes the resultant pressure drop while still providing sufficient heat transfer. It is presumed that this value must be sufficiently high, but it is not well understood precisely how high it should be for given scenarios or how the regenerator thermal mass ratio influences the performance and efficiency of Stirling engines. An extensive analysis was conducted to determine how the regenerator thermal mass ratio influences the effectiveness of single and discretized regenerators, as well as Stirling engine efficiency. The results of this study will enable Stirling engine designers to select a value of regenerator thermal mass ratio that will enhance regenerator effectiveness and Stirling engine efficiency.

In this work, a heat transfer model of a Stirling engine regenerator determined that the sole parameter that governs its effectiveness is the regenerator thermal mass ratio. The model assumes local thermal equilibrium within the regenerator and working fluid, thus neglecting local conduction heat transfer within each medium. We found good agreement between the experiments and heat transfer model, and therefore the assumptions were validated. The heat transfer model was then subjected to cyclical temperature oscillations between target temperatures, $T_{H}$ and $T_{L}$, simulating the conditions that a regenerator would be exposed to during engine operation. The regenerator thermal mass ratio was varied to observe how it influences the effectiveness of regenerators composed of a single regenerator, multiple sub-regenerators, and the efficiency of Stirling engines. The results of this study showed that as the regenerator thermal mass ratio increases, the regenerator effectiveness increases up to a limit, which then became purely dependent on the number of sub-regenerators that the regenerator is comprised of. The results also showed that an increasing regenerator thermal mass ratio corresponds to increasing Stirling engine efficiency, and multiple sub-regenerators are required to exceed $50 \%$ effectiveness, which is the maximum that a single regenerator can attain. By incorporating the influence of the regenerator thermal mass ratio in the Stirling engine's efficiency, it was observed that the engine's efficiency was not only a function of target temperatures, $T_{H}$ and $T_{L}$, but also a function of the compression ratio, the properties of the employed working fluid, the regenerator thermal mass ratio, and the number of sub-regenerators. As a result of this study, Stirling engine designers will be able to select a value of each of the aforementioned parameters to augment the Stirling engine's efficiency. 